$5-11-2012$

\title{
Information Transfer by Vector Spin Chirality in Finite Magnetic Chains
}

Matthias Menzel

Universität Hamburg

Yuriy Mokrousov

Peter Grünberg Institut and Institute for Advanced Simulation

Robert Wieser

Universität Hamburg

Jessica E. Bickel

Cleveland State University, j.e.bickel@csuohio.edu

Elena Vedmedenko

Universität Hamburg

Follow this and additional works at: https://engagedscholarship.csuohio.edu/sciphysics_facpub

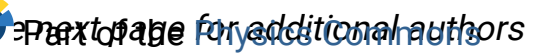

How does access to this work benefit you? Let us know!

Publisher's Statement

(C)2013 American Physical Society

\section{Repository Citation}

Menzel, Matthias; Mokrousov, Yuriy; Wieser, Robert; Bickel, Jessica E.; Vedmedenko, Elena; Blügel, Stefan; Heinze, Stefan; von Bergmann, Kirsten; Kubetzka, André; and Wiesendanger, Roland, "Information Transfer by Vector Spin Chirality in Finite Magnetic Chains" (2012). Physics Faculty Publications. 196.

https://engagedscholarship.csuohio.edu/sciphysics_facpub/196

This Article is brought to you for free and open access by the Physics Department at EngagedScholarship@CSU. It has been accepted for inclusion in Physics Faculty Publications by an authorized administrator of

EngagedScholarship@CSU. For more information, please contact library.es@csuohio.edu. 


\section{Authors}

Matthias Menzel, Yuriy Mokrousov, Robert Wieser, Jessica E. Bickel, Elena Vedmedenko, Stefan Blügel, Stefan Heinze, Kirsten von Bergmann, André Kubetzka, and Roland Wiesendanger 


\title{
Information Transfer by Vector Spin Chirality in Finite Magnetic Chains
}

\author{
Matthias Menzel, ${ }^{1}$ Yuriy Mokrousov, ${ }^{2}$ Robert Wieser, ${ }^{1}$ Jessica E. Bickel, ${ }^{1}$ Elena Vedmedenko, ${ }^{1}$ Stefan Blügel, ${ }^{2}$ \\ Stefan Heinze, ${ }^{3}$ Kirsten von Bergmann, ${ }^{1}$ André Kubetzka, ${ }^{1}$ and Roland Wiesendanger ${ }^{1}$ \\ ${ }^{1}$ Institut für Angewandte Physik, Universität Hamburg, Jungiusstr. 11, 20355 Hamburg, Germany \\ ${ }^{2}$ Peter Grünberg Institut and Institute for Advanced Simulation, Forschungszentrum Jülich, D-52425 Jülich, Germany \\ ${ }^{3}$ Institut für Theoretische Physik und Astrophysik, Christian-Albrecht-Universität zu Kiel, Leibnizstr. 15, 24098 Kiel, Germany \\ (Received 29 November 2011; published 7 May 2012)
}

\begin{abstract}
Vector spin chirality is one of the fundamental characteristics of complex magnets. For a onedimensional spin-spiral state it can be interpreted as the handedness, or rotational sense of the spiral. Here, using spin-polarized scanning tunneling microscopy, we demonstrate the occurrence of an atomicscale spin spiral in finite individual bi-atomic Fe chains on the $(5 \times 1)-\operatorname{Ir}(001)$ surface. We show that the broken inversion symmetry at the surface promotes one direction of the vector spin chirality, leading to a unique rotational sense of the spiral in all chains. Correspondingly, changes in the spin direction of one chain end can be probed tens of nanometers away, suggesting a new way of transmitting information about the state of magnetic objects on the nanoscale.
\end{abstract}

The concept of the vector spin chirality (VSC) in an ensemble of spins proved to be remarkably fruitful in the fields of magnetism of frustrated systems [1-3] and multiferroics [4], where it is naturally coupled to the electric polarization [5]. For a one-dimensional spin spiral the VSC is given by $\boldsymbol{\kappa}_{i, i+1} \propto \mathbf{S}_{i} \times \mathbf{S}_{i+1}$ with the spins $\mathbf{S}_{i}$ and $\mathbf{S}_{i+1}$ at neighboring sites $i$ and $i+1$, and it can be directly related to the spin current flowing between this pair of noncollinear spins [5]. Such a spin current transmits information about the direction of $\mathbf{S}_{n}$ to any other atom $m$ of the chain and thereby defines the direction of $\mathbf{S}_{m}$, which can then be read out. A finite one-dimensional spin spiral can therefore be used to efficiently probe and transmit information about the magnetic state of even an atomic-scale object when it interacts with such an object at one end. Practical realization of this scenario for information transport would be of great benefit, since it employs only the spin degree of freedom and no flow of charge is necessary. There are important advantages of using a system with a nonzero VSC over a chain with collinear magnetic order [6,7]: First, the VSC is a more robust quantity than the local magnetization with respect to temperature and quantum fluctuations typical for one-dimensional systems [8]. Further, a spin-spiral state is expected to be much less sensitive to parasitic magnetic stray fields always present in a device, and it would not be accompanied by creation of domain walls, harmful for efficient restructuring of the spin state. From the point of view of technological applications, the proposed way of information processing would open new paradigms in spintronic-based devices.

In order to realize this visionary goal, we have chosen an appropriate model-type system, namely, bi-atomic $\mathrm{Fe}$ chains with a length of up to $30 \mathrm{~nm}$, which form by selfassembly on a $(5 \times 1)$-reconstructed $\operatorname{Ir}(001)$ surface [9]. They appear as single strands in scanning tunneling microscopy (STM) images, reflecting the very small distance between the pairs of $\mathrm{Fe}$ atoms perpendicular to the chain axis (see Fig. 1) [11]. As shown in Fig. 2(a) in a spin-polarized (SP) STM image [12] in zero external magnetic field, the chains appear featureless. The same observation is made for spin-averaged STM measurements using nonmagnetic $\mathrm{W}$ tips not only at $B=0 \mathrm{~T}$ but also in an external magnetic field [11]. This uniform appearance of

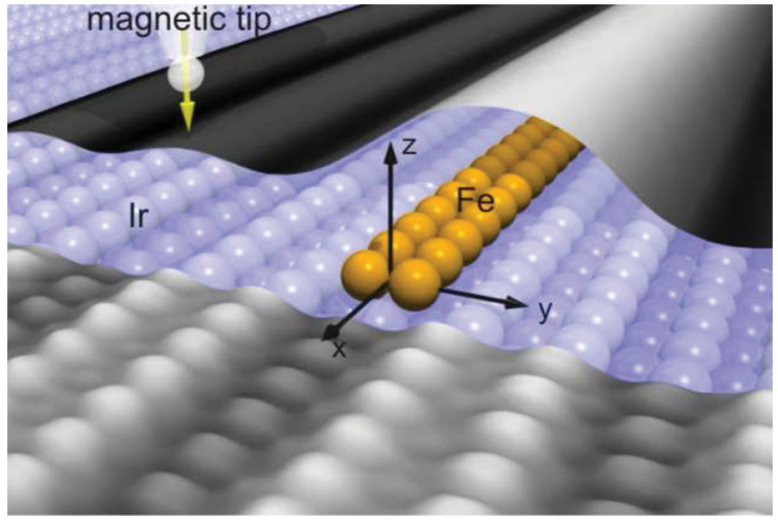

FIG. 1 (color). Schematic representation of the investigated sample system and the experimental setup. The STM image in the front shows the atomically resolved $(5 \times 1)$-reconstructed $\operatorname{Ir}(001)$ surface (measurement parameters: $U=-2 \mathrm{mV}$, $I=40 \mathrm{nA}$, room temperature). Fe atoms deposited at room temperature form bi-atomic chains in the trenches of the reconstruction (ball model), which appear as a single strand in an STM image as shown in the overlaid image at the top (measurement parameters: $U=+400 \mathrm{mV}, I=5 \mathrm{nA}$, $T=8 \mathrm{~K}$ ). The experimental setup allows to apply magnetic fields perpendicular to the sample surface, i.e., along the $z$ axis [10]. To investigate the magnetic order of the Fe chains we use spin-polarized STM with magnetic tips. 

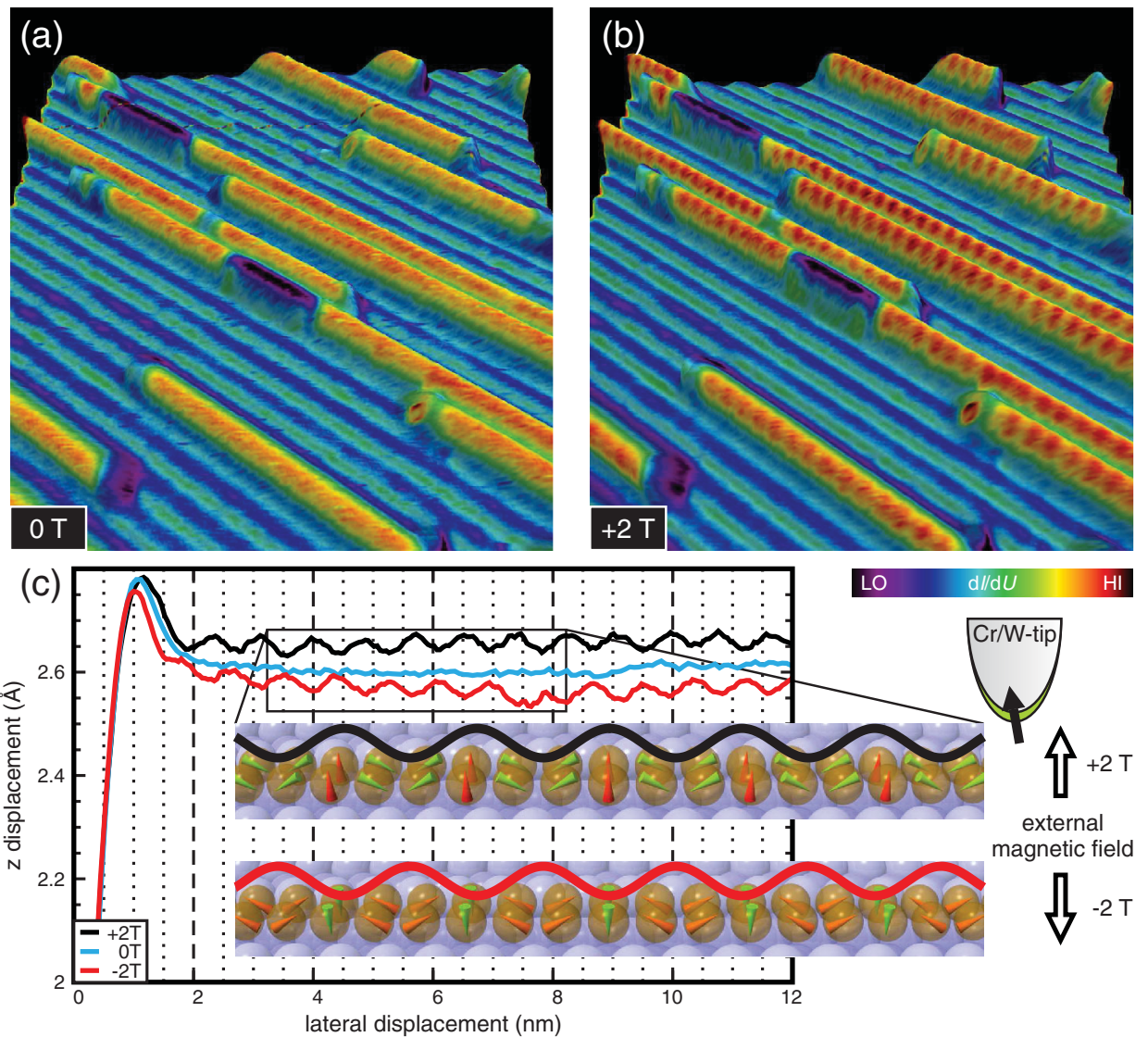

FIG. 2 (color). SPSTM measurements of Fe chains on $\operatorname{Ir}(001)$. (a) and (b) Typical sample area of $30 \times 30 \mathrm{~nm}^{2}$ measured with an Fecoated $\mathrm{W}$ tip without and with an applied external magnetic field of $B=+2 \mathrm{~T}$ perpendicular to the sample surface, respectively, (constant current images colorized with simultaneously acquired $d I / d U$ maps, measurement parameters: $U=+500 \mathrm{mV}, I=5 \mathrm{nA}$, $T=8 \mathrm{~K}$ ). (c) Topographic line profiles of the same Fe chain at $B=0 \mathrm{~T}$ and $B= \pm 2 \mathrm{~T}$ measured with a Cr-coated tip. The insets show schematically the tip magnetization and how a $120^{\circ}$ spin spiral, which is inverting in opposite fields, could explain the experimental results.

the chains changes significantly when examined with a magnetic tip in an external magnetic field: all the chains, regardless of their length, exhibit a modulation along their axes with a periodicity of three atomic distances [see Fig. 2(b)]. This image is obtained using a tip sensitive to the out-of-plane component of the magnetization. Maxima and minima along the chain axis therefore represent areas with magnetization components pointing up or down with respect to the surface [13]. The modulation persists over a wide range of applied bias voltages without changing its periodicity and could be explained by a spin-spiral state with an angle of $120^{\circ}$ between the magnetic moments of neighboring atoms along the chain axis, as it is sketched in Fig. 2(c). The periodic contrast is reversed by inverting the external field direction [Fig. 2(c)] when a Cr-coated tip is used for imaging. Because of the antiferromagnetic ordering of the Cr-coated tip, its magnetization direction does not change when applying magnetic fields of this strength [14]. Thus, the inversion of magnetic contrast along the chain axis can only be explained when the spin-spiral structure of the $\mathrm{Fe}$ chains exhibits a net magnetic moment that aligns with the magnetic field. When turning off the magnetic field the modulation vanishes and the chains again appear featureless [blue line in Fig. 2(c)].

In order to verify whether the proposed spin spiral is the magnetic ground state of bi-atomic Fe chains on $\operatorname{Ir}(001)$, we performed density functional theory (DFT) calculations [11]. We scan the magnetic phase space by calculating flat spin spirals which are the general solution of the classical Heisenberg model $E_{H}=-\sum_{i j} J_{i j} \mathbf{S}_{i} \cdot \mathbf{S}_{j}$ for a periodic lattice with the exchange constant $J_{i j}$ between the spins $\mathbf{S}_{i}$ and $\mathbf{S}_{j}$ at the atomic sites $i$ and $j$. Such a spin spiral, propagating along the chain, is given by $\mathbf{S}_{i}=S(\cos (q a i), 0, \sin (q a i))$, where $a$ is the lattice constant and $\mathbf{q}=(q, 0,0)$ is the characteristic spin-spiral vector. Varying $q$ from $q=0$ (ferromagnetic (FM) state) to $q= \pm 0.5 \frac{2 \pi}{a}$ [antiferromagnetic (AFM) state], we cover all possible spin spirals and calculate the spin-spiral dispersion energy $E(q)$ (blue dots in Fig. 3). Positive and negative values of $q$ denote clockwise and counter-clockwise spin spirals, respectively, and the dispersion is symmetric with respect to $\pm q$, as expected from the Coulomb interaction. 


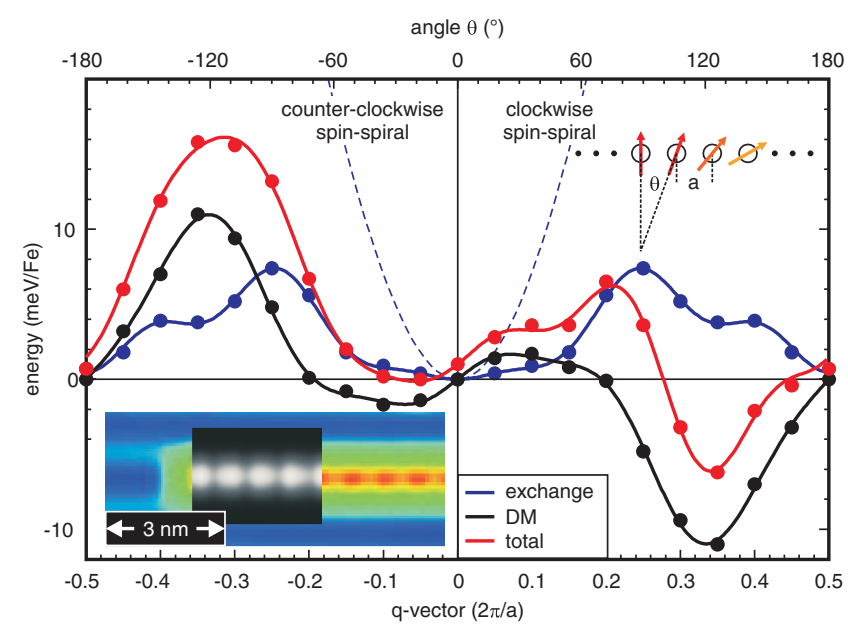

FIG. 3 (color). DFT calculations and comparison to experimental findings. Spin-spiral dispersion from first-principles calculations: contributions from Heisenberg exchange (blue dots) and Dzyaloshinskii-Moriya interaction (black dots) and their sum (red dots). The lines represent fits to the calculations with an extended Heisenberg model and the dashed blue line is the Heisenberg exchange dispersion of a freestanding bi-atomic Fe chain. Inset: comparison of a simulated SPSTM image of a $120^{\circ}$ spin spiral (gray scale inset; $5 \AA$ above the surface, $50 \%$ spin polarization of the tip) with experimental data (color; constant current image, measurement parameters: $U=$ $+300 \mathrm{mV}, I=5 \mathrm{nA}, T=8 \mathrm{~K})$.

We find that the FM solution is most favorable among all states. However, the strong FM exchange found in freestanding bi-atomic $\mathrm{Fe}$ chains (see blue dashed line, FM state $\approx 75 \mathrm{meV} / \mathrm{Fe}$ atom below the AFM state [15]) is almost completely quenched. This is due to the strong hybridization with the Ir substrate resulting in a difference between the FM and the AFM state of only $\approx 1 \mathrm{meV} / \mathrm{Fe}$ atom.

Since the exchange interactions in these bi-atomic Fe chains are very small, we anticipate a strong influence of the spin-orbit interaction (SOI) on the spin-spiral dispersion as proposed in Ref. [16]. The SOI gives rise to the magnetic anisotropy energy (MAE) $E_{\text {ani }}=\sum_{i} K_{i} \sin ^{2} \varphi_{i}$ with the anisotropy constant $K_{i}$ and the angle $\varphi_{i}$ between the spin $\mathbf{S}_{i}$ and the easy axis at site $i$. The easy axis of the Fe spin moments is out-of-plane ( $z$ axis in Fig. 1) and about $2 \mathrm{meV} / \mathrm{Fe}$ atoms lower in energy than the two high-symmetry in-plane directions. Additionally, the SOI induces the antisymmetric Dzyaloshinskii-Moriya (DM) interaction with $E_{\mathrm{DM}}=\sum_{i, j} \mathbf{D}_{i j} \cdot\left(\mathbf{S}_{i} \times \mathbf{S}_{j}\right)$, where $\mathbf{D}_{i j}$ is the DM vector. At surfaces a nonvanishing $E_{\mathrm{DM}}$ is always possible due to inversion-asymmetry [17]. If the DM interaction can compete with the Heisenberg exchange it can give rise to a unique direction of the VSC. Based on symmetry arguments, $\mathbf{D}_{i j}$ and thus the VSC aligns along the $y$ axis, favoring cycloidal spin spirals with a unique rotational sense where the magnetic moments rotate in the $x z$ plane (cf. Fig. 1) [18-20].
We evaluate the correction to $E(q)$ due to the DM interaction, $E_{\mathrm{DM}}(q)$, from DFT [11]. The results for $E_{\mathrm{DM}}(q)$ are presented as black dots in Fig. 3, and show that the magnitude of $E_{\mathrm{DM}}(q)$ competes with the contribution from the Heisenberg exchange, reaching as much as $10 \mathrm{meV}$. Summing up Heisenberg and DM contributions and including an energy shift due to the MAE, we find a robust cycloidal spin-spiral ground state, several meV below the FM state, in the vicinity of $q \approx+1 / 3 \frac{2 \pi}{a}$ (red dots). This value and sign of the spin-spiral vector corresponds to a clockwise $120^{\circ}$ spin spiral, running along the chain axis. Owing to the antisymmetric nature of $E_{\mathrm{DM}}(q)$ with respect to $\pm q$, the counter-clockwise spin-spiral state with $q=-(1 / 3) \frac{2 \pi}{a}$ is much higher in energy, which manifests the unique rotational sense of our spin structure [20] described by a unique VSC that points along the $y$ axis for all chains. Remarkably, the magnetic unit cell of this $120^{\circ}$ state consists of three $\mathrm{Fe}$ atoms along the chain axis, which is the minimal periodicity necessary to form a noncollinear state. As shown in Fig. 3, a simulated SPSTM image of such a state based on the DFT calculations is in excellent agreement with the experimental findings.

The first-principles calculations correspond to a situation of Fe chains at zero temperature and zero magnetic field, and in order to understand the influence of temperature and magnetic field on our system we use a heat-bath Monte Carlo (MC) method [21] employing an extended Heisenberg model $E_{\mathrm{tot}}=E_{H}+E_{\mathrm{ani}}+E_{\mathrm{DM}}+E_{B}$ including the effect of an out-of-plane magnetic field $E_{B}=$ $-\mu_{s} B \sum_{i} S_{i}^{z}$, where $\mu_{s}$ is the magnetic moment. The material parameters $J_{i j}$ and $\mathbf{D}_{i j}$ are obtained from fits to the first-principles calculations (see blue and black line in Fig. 3). To capture the nontrivial dispersions of $E(q)$ and $E_{\mathrm{DM}}(q)$, we included the $J_{i j}$ and $\mathbf{D}_{i j}$ parameters up to six nearest neighbors along the chain axis, which are listed in the Supplemental Material [11]. The coupling between the pair of Fe atoms along the $y$ axis is FM with $J_{\text {perp }} \approx$ $160 \mathrm{meV} / \mathrm{Fe}$ atom and the spin moment of each $\mathrm{Fe}$ atom and the MAE were set to their respective DFT values of $\mu_{s}=2.75 \mu_{B}$ and $K_{i}=2 \mathrm{meV}$, irrespective of the position of the atom within the finite chain. Since the Fe chains observed in the experiments show a broad length distribution ranging from only a few $\mathrm{nm}$, corresponding to $\approx 10$ pairs of atoms, up to several tens of nm, corresponding to up to 150 pairs, we chose to investigate a representative chain consisting of 30 pairs of atoms. The value of the time-averaged magnetization $M_{z}$ in our MC simulations is normalized, meaning that a value of one corresponds to a nonfluctuating magnetic moment, while a smaller value indicates the presence of thermal fluctuations. The analysis of the time-averaged out-of-plane magnetization $M_{z}$ of the entire chain at zero field shows that it is negligible for all temperatures, see Fig. 4(a), explaining the lack of magnetic contrast in our experiments at $B=0 \mathrm{~T}$. At the measurement temperature of $8 \mathrm{~K}$ a single snapshot of the $x, y$, and $z$ 


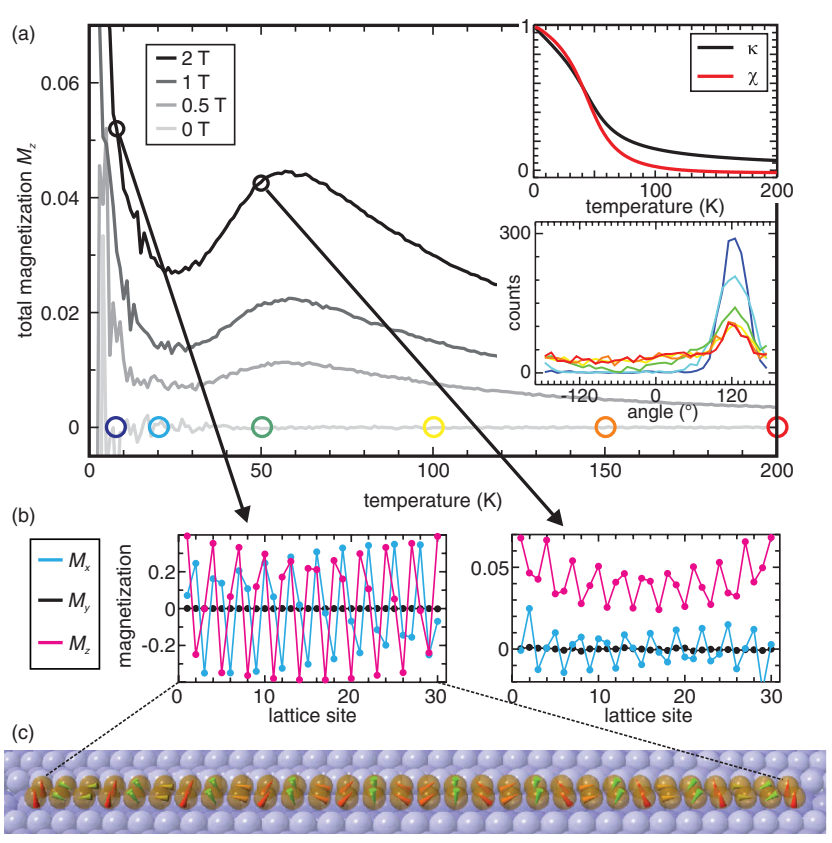

FIG. 4 (color). Monte-Carlo simulations for a 30 atom long chain. (a) Temperature dependence of $M_{z}$ for different external magnetic fields applied along the $z$ axis. Upper inset depicts the temperature dependence of the normalized chiral and scalar order parameters as function of temperature. Lower inset displays the distribution of angles between nearest neighbor spins along the chain (the color of the lines corresponds to the temperature of the system given by the circle of the corresponding color below). (b) Laterally resolved time-averaged magnetization for two different temperatures at $B=+2 \mathrm{~T}$. (c) Threedimensional representation of magnetization components of every atom for $T=8 \mathrm{~K}$ and $B=+2 \mathrm{~T}$.

component of the magnetization along the chain reveals a $120^{\circ}$ clockwise spin spiral, except for small deviations in the angles, but rapid switching of this spin spiral results in a vanishing time-averaged $M_{z}$.

In order to take a deeper look into the intrinsic spin ordering of the $\mathrm{Fe}$ chains at $B=0 \mathrm{~T}$ as a function of temperature, we calculate the normalized chiral and scalar order parameters, $\kappa=\frac{1}{\sin \theta_{0}}\left\langle\left(\mathbf{S}_{i} \times \mathbf{S}_{i+1}\right)_{y}\right\rangle$ and $\chi=$ $\frac{\left\langle\arccos \left(\mathbf{S}_{i} \cdot \mathbf{S}_{i+1}\right)\right\rangle-\theta_{\infty}}{\theta_{0}-\theta_{\infty}}\left(\theta_{0}=122.4^{\circ}\right.$ and $\theta_{\infty}=90^{\circ}$ are the angles of the spin configuration at zero and at infinite temperatures), obtained from time- and chain-averaged VSC and scalar product between neighboring spins, respectively [22]. For low temperatures, see Fig. 4(a) upper inset, both $\kappa$ and $\chi$ are close to 1, which corresponds to an almost ideal clockwise $120^{\circ}$ spin-spiral state at any time. With increasing temperature the local fluctuations of the spin moments of each atom become more and more pronounced, which results in a state with $\chi \approx 0$ at temperatures above $100 \mathrm{~K}$. Surprisingly, at this temperature the chiral order parameter $\kappa$ is still far from zero, and it decays much more slowly with temperature. This means that the chiral correlation between the spins is still present for $T \gg 100 \mathrm{~K}-$ a situation reminiscent of that for the vector spin chiral liquid state, predicted to occur in onedimensional spin systems [22-25] and observed experimentally in the quasi-one-dimensional molecular helimagnetic compound $\mathrm{Gd}(\mathrm{hfac})_{3}$ NITEt [8]. It can be understood by looking at the time- and chain-averaged distribution of the angle between the nearest neighbor spins in the $x z$ plane as a function of temperature, lower inset of Fig. 4(a). Despite the fact that the averaged nearest-spin scalar product becomes negligible very quickly, the noticeable preference of the spin's fluctuations with respect to its neighbor towards positive angles remains for a very wide range of temperatures. One can imagine that, under conditions of a sufficiently strong VSC, such asymmetry can be used to obtain information about the spin's fluctuations on one end of the chain by monitoring the dynamics of the spin at the other end, even at comparatively high temperatures.

At finite $B$ an out-of-plane magnetization $M_{z}$ of the chain arises, see Fig. 4(a), and at the experimental conditions of $T=8 \mathrm{~K}$ and $B=2 \mathrm{~T}$ we find a time-averaged magnetization of the atoms in the chain as displayed in Fig. 4(b) (left) and (c), in good agreement with the SPSTM measurements. The external magnetic field can induce a net moment of the chain, e.g., owing to the larger susceptibility of the end spins which have a reduced coordination number, thus facilitating a preferred orientation of the spin spiral within the chain. Such a finite size mechanism appears to be crucial for the experimental observation of the noncollinear magnetic state of our chains, since for an infinite chain with the same parameters, the averaged local magnetization as determined by the MC simulations is always zero at any temperature, even for very large magnetic fields. For our 30 atom long chain in Fig. 4(a) at low $T$ the net moment is preferentially oriented along the magnetic field, resulting in a comparably large value of $M_{z}$. With increasing $T$ this value decreases due to thermal fluctuations. For even larger $T$ the spin-spiral order is weakened and all spins have components parallel to the external field, thus contributing to $M_{z}$; see Fig. 4(b). Above the transition temperature of roughly $60 \mathrm{~K}$ the magnetic order is destroyed by thermal fluctuations.

Finally, we demonstrate that the suggested novel mechanism of information transport based on VSC can be realized in this type of system by attaching the Fe chain to a stable magnetic particle. Figure 5 shows an SPSTM image at $B=$ $0 \mathrm{~T}$ of one isolated Fe chain (bottom left) and one Fe chain coupled to a ferromagnetic Co chain (center). The isolated chain does not show the periodicity indicative of spin-spiral order due to rapid thermal switching [cf. Fig. 2(a)]. In contrast, the Fe chain that is attached to the Co chain displays a distinct magnetic signal. This stabilization of the spin spiral happens due to strong direct exchange interaction of the end Fe spins with the Co chain, which fixes their direction in space. With the magnetization of one chain end fixed in 

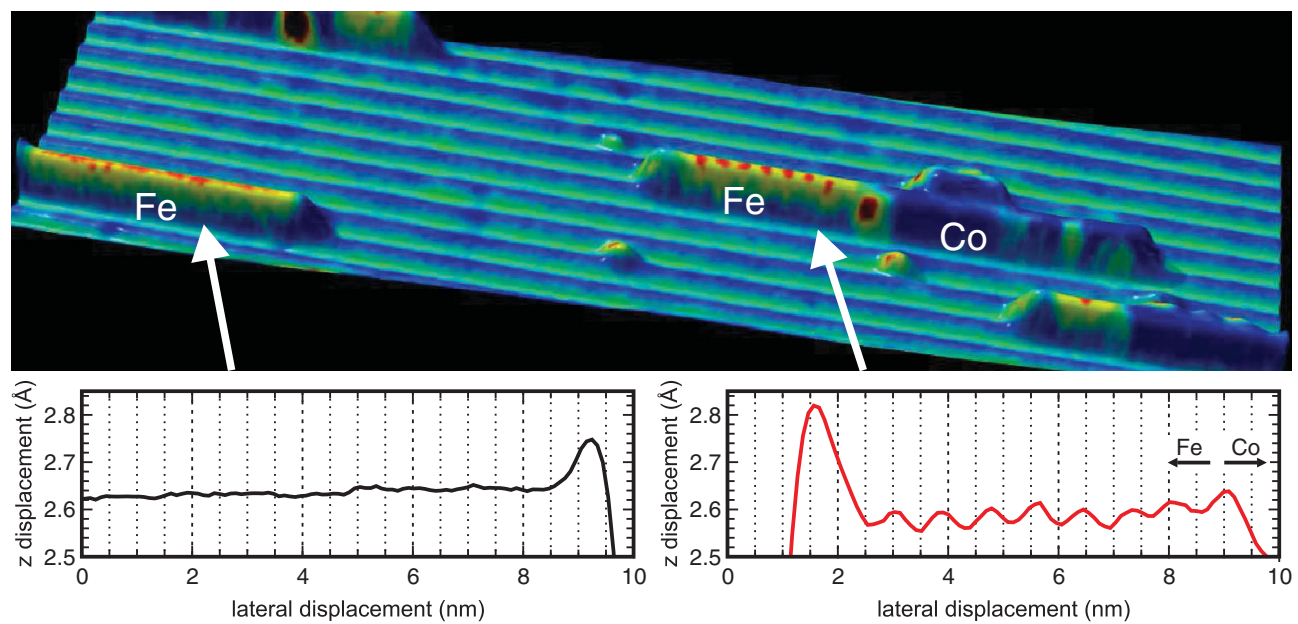

FIG. 5 (color). SPSTM measurement of an isolated Fe chain (left) and an Fe chain directly connected to a ferromagnetic Co chain (center) taken at $B=0 \mathrm{~T}$ with a Cr bulk tip $\left(50 \times 12.5 \mathrm{~nm}^{2}\right.$ constant current image, colorized with $d I / d U$-map, measurement parameters: $U=+500 \mathrm{mV}, I=2 \mathrm{nA}, T=8 \mathrm{~K}$ ). As seen in both the image and the line profiles below, the isolated Fe chain appears featureless while the one attached to the Co shows the periodicity indicative of spin-spiral order.

space and time, the intrachain coupling leads to a stabilization of the whole Fe chain. Correspondingly, by measuring the direction of any Fe spin, even nanometers away, we can 'read out' the state of the Co chain and monitor its changes, owing to the link by the vector spin chirality.

We thank Y. Yoshida, S. Schröder, D. S. G. Bauer, Ph. Mavropoulos, B. Zimmermann, M. Heide, W. Selke, and G. Bihlmayer for fruitful discussions. M. M., R. Wieser, J.E. B., E. V., K. v. B., A. K., R. Wiesendanger gratefully acknowledge financial support from the DFG via SFB668, from the EU via the ERC Advanced Grant FURORE, and from the Cluster of Excellence NANOSPINTRONICS funded by the Forschungs- und Wissenschaftsstiftung Hamburg. J.E. B. also acknowledges financial support from the Alexander von Humboldt Foundation. Y. M. acknowledges the Jülich Supercomputing Centre and funding under the HGF-YIG Programme VH-NG-513 and S. H. thanks the DFG for financial support under Grant No. HE3292/8-1

[1] J. Villain, J. Phys. C 10, 4793 (1977).

[2] D. Grohol, K. Matan, J.-H. Cho, S.-H. Lee, J. W. Lynn, D. G. Nocera, and Y. S. Lee, Nature Mater. 4, 323 (2005).

[3] X. G. Wen, F. Wilczek, and A. Zee, Phys. Rev. B 39, 11413 (1989).

[4] S.-W. Cheong and M. Mostovoy, Nature Mater. 6, 13 (2007).

[5] H. Katsura, N. Nagaosa, and A. V. Balatsky, Phys. Rev. Lett. 95, 057205 (2005).

[6] C. F. Hirjibehedin, C. P. Lutz, and A. J. Heinrich, Science 312, 1021 (2006).

[7] A. A. Khajetoorians, J. Wiebe, B. Chilian, and R. Wiesendanger, Science 332, 1062 (2011).
[8] F. Cinti, A. Rettori, M. G. Pini, M. Mariani, E. Micotti, A. Lascialfari, N. Papinutto, A. Amato, A. Caneschi, D. Gatteschi, and M. Affronte, Phys. Rev. Lett. 100, 057203 (2008).

[9] L. Hammer, W. Meier, A. Schmidt, and K. Heinz, Phys. Rev. B 67, 125422 (2003).

[10] O. Pietzsch, A. Kubetzka, D. Haude, M. Bode, and R. Wiesendanger, Rev. Sci. Instrum. 71, 424 (2000).

[11] See Supplemental Material at http://link.aps.org/ supplemental/10.1103/PhysRevLett.108.197204 for details.

[12] R. Wiesendanger, Rev. Mod. Phys. 81, 1495 (2009).

[13] D. Wortmann, S. Heinze, P. Kurz, G. Bihlmayer, and S. Blügel, Phys. Rev. Lett. 86, 4132 (2001).

[14] O. Pietzsch, A. Kubetzka, M. Bode, and R. Wiesendanger, Phys. Rev. Lett. 92, 057202 (2004).

[15] Y. Mokrousov, A. Thiess, and S. Heinze, Phys. Rev. B 80, 195420 (2009).

[16] R. Mazzarello and E. Tosatti, Phys. Rev. B 79, 134402 (2009).

[17] M. Bode, M. Heide, K. von Bergmann, P. Ferriani, S. Heinze, G. Bihlmayer, A. Kubetzka, O. Pietzsch, S. Blügel, and R. Wiesendanger, Nature (London) 447, 190 (2007).

[18] I. Dzyaloshinskii, J. Phys. Chem. Solids 4, 241 (1958).

[19] T. Moriya, Phys. Rev. 120, 91 (1960).

[20] M. Heide, G. Bihlmayer, Ph. Mavropoulos, A. Bringer, and S. Blügel, Psi-k Highlight 78, 1 (2006).

[21] R. Wieser, E. Y. Vedmedenko, and R. Wiesendanger, Phys. Rev. B 77, 064410 (2008).

[22] F. Cinti, A. Cuccoli, and A. Rettori, Phys. Rev. B 83, 174415 (2011).

[23] T. Hikihara, L. Kecke, T. Momoi, and A. Furusaki, Phys. Rev. B 78, 144404 (2008).

[24] J. Sudan, A. Lüscher, and A. M. Läuchli, Phys. Rev. B 80, 140402 (2009).

[25] S. Furukawa, M. Sato, and S. Onoda, Phys. Rev. Lett. 105, 257205 (2010). 